\title{
Optical Characterization of $\mathrm{LiNbO}_{3}$ Whispering Gallery Mode Micro- Resonators Fabricated by Surface Tension Reshaping
}

\author{
C. Y. J. Ying ${ }^{1}$, G. S. Murugan ${ }^{1}$, G. Brambilla ${ }^{1}$, C. L. Sones ${ }^{1}$, E. Soergel ${ }^{2}$, \\ J. S. Wilkinson ${ }^{1}$, R. W. Eason ${ }^{1}$, M. N. Zervas ${ }^{1}$, S. Mailis ${ }^{1}$ \\ 1. Optoelectronics Research Centre, University of Southampton, Highfield, Southampton, SO17 1BJ, United Kingdom \\ 2. Institute of Physics, University of Bonn, Wegelerstr. 8, 53115 Bonn, Germany
}

Lithium niobate (LN) is an attractive material for high-Q whispering gallery mode (WGM) resonators due to its wide optical transparency window, high electro-optic coefficient and nonlinearity. Recently, LN structures suitable for WGM micro-resonators have been fabricated using surface tension reshaping of a previously microstructured substrates, producing ultra-smooth surfaces while also maintaining the useful crystalline properties of the original material [1]. The method is based on the preferential melting of a surface layer [2] at temperatures close to the melting point for the bulk material. Upon cooling, the melted surface layer re-crystallizes, seeded by the bulk crystal that remains solid during the process, and is reshaped by the surface tension to form ultrasmooth single crystal superstructures. Fig. 1 shows some surface tension reshaped structures which have been obtained by varying the initial microstructure and the thermal treatment conditions, (a): $5 \mu \mathrm{m}$ prolate spheroid, (b): $3 \mu \mathrm{m}$ capsule, (c-e): $7,30,80 \mu \mathrm{m}$ diameter pillars. Such structures are suitable for supporting WGMs as the smooth side surface should lead to low scattering loss. Their obtainable small dimensions leads to a small number of supported WGMs and increases the free spectral range (FSR). Both are beneficial for the fabrication of spectral filters and lasers.

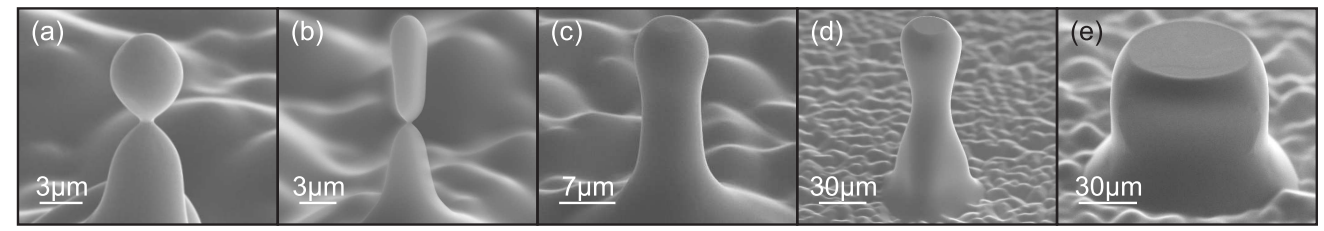

Fig. 1 SEM images tilted by $60^{\circ}$ showing a variety of achieved surface tension reshaped structures.

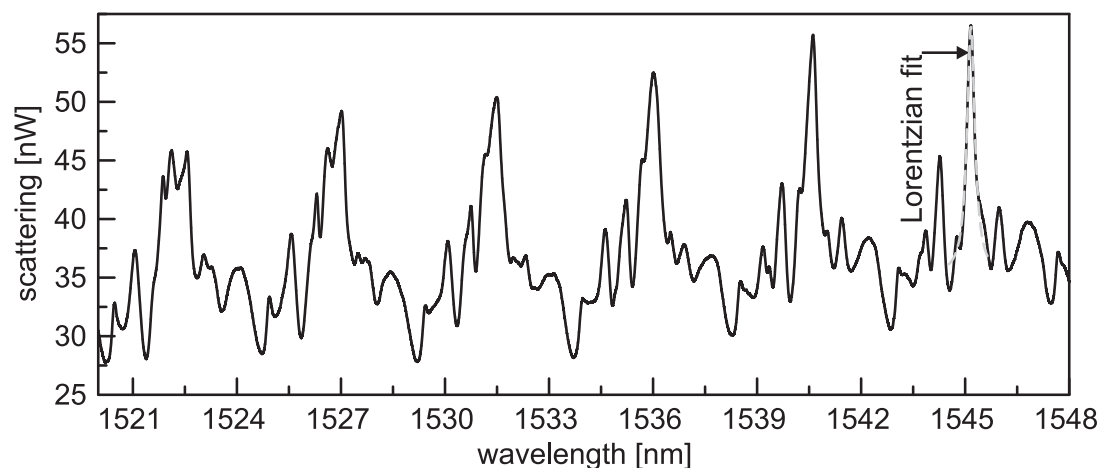

Fig. 2 Scattered signal with fitted Lorentzian used for the determination of $\mathrm{Q}$ factors of the microresonator.

Preliminary optical characterisation of the microresonator in Fig. 1e has been conducted. A U-shaped silica fibre taper with a waist diameter of about $2 \mu \mathrm{m}$ was used to couple light in and out of the microresonator. It was observed that when approaching the resonator the fibre taper is attracted by the surface of the structure and loads the cavity. A tunable laser source was used to observe the resonances of the WGM structure. In order to investigate the resonances the light transmitted through the fibre taper and the light scattered from the resonator were monitored during the wavelength scanning process. The scattered light spectrum in Fig. 2 shows a sequence of resonances. A $\mathrm{Q}$ factor of 5600 has been calculated by fitting a Lorentzian curve to one of the peaks in the spectrum shown in Fig. 2, which has a corresponding FSR of 4.55nm. The shape of the resonator (Fig. 1e) resembles a truncated microbottle [3]. Such open resonators are known to leak from their two ends [4], and the loss depends on the curvature of the micro-resonator. Leakage from the resonator's base was experimentally observed. It is expected that higher $\mathrm{Q}$ factors will be measured in microresonators with larger curvature.

\section{References}

[1] C. Y. J. Ying, C. L. Sones, A. C. Peacock, F. Johann, E. Soergel, R. W. Eason, M. N. Zervas, and S. Mailis, "Ultra-smooth lithium niobate photonic micro-structures by surface tension reshaping," Opt. Express 18, 11508-11513 (2010).

[2] R. Lipowsky, "Surface-induced disorder and surface melting," in Springer Proc. in Physics, 1990), 158-166.

[3] G. S. Murugan, J. S. Wilkinson, and M. N. Zervas, "Selective excitation of whispering gallery modes in a novel bottle microresonator,"

Opt. Express 17, 11916-11925 (2009).

[4] G. S. Murugan, J. S. Wilkinson, and M. N. Zervas, "Optical excitation and probing of whispering gallery modes in bottle microresonators: potential for all-fiber add-drop filters," Opt. Lett. 35, 1893-1895 (2010). 